ORIGINAL ARTICLE

\title{
Frequency of feto-maternal complications in patients with intrahepatic cholestasis of pregnancy.
}

\author{
Arooj Akram1, Joveria Sadaf ${ }^{2}$, Asia Aziz ${ }^{3}$, Sana Ara ${ }^{4}$, Tanzila Rafiq ${ }^{5}$, Aslam Mahmood Malik
}

Article Citation: Akram A, Sadaf J, Aziz A, Ara S, Rafiq T, Malik AM. Frequency of feto-maternal complications in patients with intrahepatic cholestasis of pregnancy. Professional Med J 2022; 29(2):212-217. https://doi.org/10.29309/TPMJ/2022.29.02.6394

ABSTRACT... Objective: To determine the frequency of complications in patients with intrahepatic cholestasis of pregnancy. Study Design: Descriptive Case Series. Setting: Department of Obstetrics \& Gynecology, Shahida Islam Medical College, Lodhran. Period: July 2019 to December 2020. Material \& Methods: A total of 141 patients with intrahepatic cholestasis of pregnancy of age 18 to 40 years were included. Patients with multiple pregnancies, history of alcohol intake, CR and preexisting chronic liver disease were excluded. All women were followed till delivery (gestational age $\leq 41$ weeks) and feto-maternal outcome i.e. postpartum hemorrhage, cesarean section, APGAR score $<7$ at 5 minutes, low birth weight, prematurity, meconium stained liquor and intrauterine fetal death (yes/no) was noted. Results: Feto-maternal outcome was as follows; postpartum hemorrhage in $18(12.77 \%)$, cesarean section in $47(33.33 \%)$, preterm birth in $34(24.11 \%)$, APGAR score $<7$ at five minutes in $13(9.22 \%)$, meconium stained liquor in $45(31.91 \%)$ patients, low birth weight in $14(9.93 \%)$ and Intra-uterine fetal death in $11(7.80 \%)$ patients. Conclusion: Our study concluded that we should develop some serious recommendations for these high risk patients in our routine practice. It will reduce the morbidity and mortality related to this high risk condition.

Key words: $\quad$ Cholestasis, Cesarean, Pregnancy, Preterm Delivery, Postpartum Hemorrhage.

\section{INTRODUCTION}

Obstetric cholestasis $(\mathrm{OC})$ or intrahepatic cholestasis of pregnancy (ICP), is a pregnancy specific liver disease, presented with intense generalized pruritus that is not associated with skin rash. ${ }^{1}$ this condition is temporary and it is characterized by increased liver enzyme (one or more) on blood tests. Though its pathophysiology is not understood ${ }^{2}$ a genetic background is suggested. The condition occurs by demographic variation and family clustering that suggests genetic involvement. It incidence is highest in Chile-Bolivia (6\%-27\%) and Sweden $(1-1.5 \%) .^{3}$ It prevalence is about 1.2 to $1.5 \%$ of women of Indian or Pakistani Asian females. ${ }^{4}$

Although relatively benign to women, ICP can profoundly compromise the quality of life by distressing pruritus with disturbed sleep, worries about the adverse fetal outcome and more frequent antenatal visits thus adding exponentially to the stress of pregnancy. Despite improved obstetric care, mothers with ICP and the caring obstetricians still face the adverse fetal outcome that includes but not limited to preterm delivery, antenatal meconium staining of the amniotic fluid with associated respiratory complications, fetal bradycardia, fetal distress and even intrauterine fetal death. ${ }^{4}$

Intrahepatic cholestasis of pregnancy constitutes an important cause of neonatal and maternal morbidity and mortality. It accounts for $60 \%$ perinatal and $14 \%$ of maternal mortality. ${ }^{5}$ ICP is linked with an increased risk of post-partum hemorrhage as a result of mal-absorption of vitamin K. ICP poses a significant risk for the fetus. ${ }^{6,7}$
1. MBBS, Post Graduate Resident, Shahida Islam Medical Complex Lodhran.

2. FCPS, Assistant Professor Obs and Gynae, Shahida Islam Medical Complex Lodhran

3. FCPS, Assistant Professor Community Medicine, Quaid e Azam Medical College Bahawalpur.

4. FCPS, Senior Registrar Obs and Gynae, Shahida Islam Medical Complex Lodhran.

5. FCPS, Assistant Professor Obs and Gynae, Shahida Islam Medical Complex Lodhran

6. FCPS, Professor and HOD Obs and Gynae, Shahida Islam Medical Complex Lodhran.

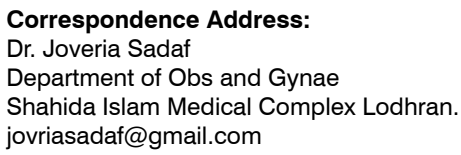

Correspondence Address:

Dr. Joveria Sadaf

Department of Obs and Gynae

Shahida Islam Medical Complex Lodhran. jovriasadaf@gmail.com

Article received on: 
The adverse effects of intrahepatic cholestasis of pregnancy on the neonate and fetus include increased risks of meconium staining of amniotic fluid, preterm birth, fetal distress leading to fetal death and fetal bradycardia. ${ }^{5,6,8,9}$ Mechanisms of poor fetal outcome are not known till date. It is found that poor fetal outcomes like asphyxia events and spontaneous preterm delivery are associated with elevated total serum bile acids ( $>40 \mu \mathrm{mol} / \mathrm{L})$ in maternal plasma. ${ }^{7}$ In a study, the feto-maternal outcome in ICP was cesarean delivery in $46.25 \%$, postpartum hemorrhage in $11.25 \%$, Apgar score $<7$ at 5 minutes in $13.75 \%$, low birth weight in $22.50 \%$, prematurity in $10.0 \%$, meconium stained liquor in $32.5 \%$ and intrauterine fetal death in $6.25 \% .^{10}$

Obstetric cholestasis is considered worldwide as an important cause offeto-maternal complications. The earlier the detection of this condition, the better is the detection of at risk fetuses, so there should be a clear plan of action and protocols for such patients that are antenatal fetal monitoring and early delivery. We have decided to conduct this study to calculate the prevalence of fetomaternal complications in ICP. This study will not only provide the local data of the problem but will also be a useful addition in the existing literature. Moreover, a proper protocol can be designed in these high risk patients for antenatal monitoring and proper management plans to reduce the fetal morbidity and mortality. Although previously studies are done on this but locally the available data is very scarce and research must be required in this regard for proper managing these particular patients and arrangements of all necessary measures for better feto-maternal outcome. The objective of the study is "To determine the prevalence of feto-maternal complications in intrahepatic cholestasis of pregnancy."

\section{MATERIAL \& METHODS}

This Descriptive case series was conducted in department of Obstetrics \& Gynecology, Shahida Islam Medical College Lodhran and Bahawal Victoria Hospital Bahawalpur from July 2019 to 31st December 2020. Sample size of 141 cases has been calculated by using WHO calculator for sample size with $95 \%$ confidence level, $4 \%$ margin of error and taking expected percentage of IUFD as $6.25 \% .10$. The Sample Technique used was Non-probability consecutive sampling.

After approval from ethical committee all women with intrahepatic cholestasis of pregnancy at gestational age $\geq 28$ weeks as assessed on LMP were included in the study i.e. patients presented with pregnancy and presence of skin pruritus (sensation or desire to scratch) and presence of anyone of the following; alanine aminotransferase $(\mathrm{ALT})>45 \mathrm{U} / \mathrm{L}$, aspartate aminotransferase (AST) $>40 \mathrm{U} / \mathrm{L}$, and fasting serum bile acids $>10 \mu \mathrm{mol} / \mathrm{L}$. Patients with multiple pregnancies (assessed on ultrasonography), patients with history of alcohol intake and chronic renal failure or liver disease were excluded. Patients having other causes of biliary obstruction i.e. gallstones confirmed on ultrasonography were also excluded.

Total of 141 women with intrahepatic cholestasis of pregnancy who were admitted to Department of Obstetrics \& Gynecology, Shahida Islam Medical Teaching Hospital, Lodhran and Bahawal Victoria Hospital Bahawalpur fulfilling the inclusion criteria were selected. Informed consent was taken from each patient and management was done according to ward protocol. All women were followed till delivery (gestational age $\leq 41$ weeks) and feto-maternal outcome i.e. postpartum hemorrhage, cesarean section, Apgar score $<7$ at 5 minutes, low birth weight, prematurity, meconium stained liquor and intrauterine fetal death (yes/no) was noted as per-operational definition.

This all data (age, gestational age, parity, BMI, gestational diabetes mellitus, pregnancy induced hypertension, place of living, postpartum hemorrhage, cesarean section, Apgar score $<7$ at 5 minutes, low birth weight, prematurity, meconium stained liquor and intrauterine fetal death) was recorded on a specially designed proforma. The collected information was analyzed by computer software SPSS version 20.0. Age, gestational age, parity and BMI were presented as mean and standard deviation. Gestational diabetes mellitus, pregnancy induced hypertension, place of living, postpartum hemorrhage, cesarean 
section, Apgar score $<7$ at 5 minutes, low birth weight, prematurity, meconium stained liquor and intrauterine fetal death were presented as frequency and percentage.

Effect modifiers like age, gestational age, parity, BMI, gestational diabetes mellitus, pregnancy induced hypertension and place of living were controlled through stratification. Chi square was applied post-stratification to see their effects on outcome and p-value $\leq 0.05$ was considered as significant.

\section{RESULTS}

Table-I is showing the distribution of the patients according to Age, BMI, parity, place of living, pregnancy induced hypertension and gestational diabetes mellitus. While Table-Il shows the fetomaternal complication rate in patients with cholestasis of pregnancy.

\begin{tabular}{|c|c|c|c|c|}
\hline & & $\begin{array}{l}\text { No. of } \\
\text { Patients }\end{array}$ & \%age & $\begin{array}{l}\text { Mean } \\
\stackrel{ \pm}{\text { SD }}\end{array}$ \\
\hline \multirow{3}{*}{$\begin{array}{l}\text { Age of the } \\
\text { patient }\end{array}$} & $18-30$ & 93 & 65.96 & \multirow{2}{*}{$\begin{array}{c}27.16 \\
\pm \\
4.81 \text { year }\end{array}$} \\
\hline & $31-40$ & 48 & 34.04 & \\
\hline & Total & 141 & 100.0 & \multirow[b]{2}{*}{$\begin{array}{l}29.72 \\
\pm \\
2.92 \mathrm{~kg} / \\
\mathrm{m} 2\end{array}$} \\
\hline \multirow[t]{3}{*}{ BMI (kg/m2) } & $\leq 30$ & 84 & 59.57 & \\
\hline & $>30$ & 57 & 40.43 & \\
\hline & Total & 141 & 100.0 & \\
\hline \multirow{3}{*}{ Parity } & $0-2$ & 89 & 63.12 & \multirow{2}{*}{$\begin{array}{c}2.33 \pm \\
0.86\end{array}$} \\
\hline & $3-5$ & 52 & 36.88 & \\
\hline & Total & 165 & 100.0 & \\
\hline \multirow{3}{*}{$\begin{array}{l}\text { Place of } \\
\text { living }\end{array}$} & Rural & 75 & 53.19 & \\
\hline & Urban & 66 & 46.81 & \\
\hline & Total & 141 & 100.0 & \\
\hline \multirow{3}{*}{$\begin{array}{l}\text { Pregnancy } \\
\text { induced } \\
\text { hypertension } \\
(\mathrm{PIH})\end{array}$} & Yes & 33 & 23.40 & \\
\hline & No & 108 & 76.60 & \\
\hline & Total & 141 & 100.0 & \\
\hline \multirow{3}{*}{$\begin{array}{l}\text { Gestational } \\
\text { diabetes } \\
\text { mellitus } \\
\text { (GDM) }\end{array}$} & Yes & 23 & 16.31 & \\
\hline & No & 118 & 83.69 & \\
\hline & Total & 141 & 100.0 & \\
\hline \multicolumn{5}{|c|}{$\begin{array}{l}\text { Table-I. Distribution of patients according to Age, } \\
\text { BMI, parity, place of living, pregnancy induced } \\
\text { hypertension and gestational diabetes mellitus. }\end{array}$} \\
\hline
\end{tabular}

\begin{tabular}{|l|c|c|}
\hline \multirow{2}{*}{ Outcome } & \multicolumn{2}{|c|}{ Frequency (\%) } \\
\hline Post-partum hemorrhage & Yes & No \\
\hline Cesarean section & 18 & 123 \\
\hline Preterm delivery & $(12.77 \%)$ & $(87.23 \%)$ \\
\hline APGAR score $<7$ at five minutes & $(33.33 \%)$ & 94 \\
\hline \multirow{2}{*}{ Meconium stained liquor } & 34 & 107 \\
\hline Low birth weight & $(24.11 \%)$ & $(75.89 \%)$ \\
\hline Intra-uterine fetal death & 45 & 128 \\
\hline Table-II. Feto-maternal outcome in intrahepatic \\
\hline cholestasis of pregnancy.
\end{tabular}

\section{DISCUSSION}

ICP is a condition that has relatively less complications for the mother but for the fetus it causes serious complications like intrauterine fetal death, meconium stained liquor poor APGAR scores at birth. ${ }^{11}$ The cause behind these complications is poorly understood. First, research in animals has shown a detrimental effect of high bile acids levels on cardio-myocytes, which cause arrythmia. ${ }^{12,13}$ This fetal arrhythmia that is associated with bile acids may explain intra uterine fetal death. Second, bile acids also exert its vaso-constrictive effects on the chorionic villous blood vessels that can further explain intra uterine fetal hypoxia, fetal distress and death. ${ }^{14}$

Age range in this study was from 18 to 40 years with mean age of $27.16 \pm 4.81$ years. Majority of the patients 93 (65.56\%) were between 18 to 30 years of age. Prevalence of feto-maternal outcome was as follows; postpartum hemorrhage in $18(12.77 \%)$, cesarean section in 47 (33.33\%), preterm birth in 34 (24.11\%), APGAR score $<7$ at five minutes in $13(9.22 \%)$, meconium stained liquor in 45 (31.91\%) patients, low birth weight in 14 (9.93\%) and Intra-uterine fetal death in 11 (7.80\%) patients. In a study, the feto-maternal outcome were observed in ICP, cesarean delivery rate in $46.25 \%$, postpartum hemorrhage in $11.25 \%$, APGAR score $<7$ at 5 minutes in 
$13.75 \%$, low birth weight in $22.50 \%$, prematurity in $10.0 \%$, meconium stained liquor in $32.5 \%$ and intrauterine fetal death in $6.25 \% .{ }^{15}$

In a study, the authors found that the pregnancies that were complicated by intra hepatic cholestasis out of them $33 \%$ were complicated by the fetomaternal bad outcome, like poor APGAR scores at birth fetal hypoxia and meconium stained liquor but no intra-uterine fetal death was recorded. ${ }^{16}$

A local study was done on 1250 ladies. Total 35 cases [2.8\%] had cholestasis in pregnancy. The mean age of the women was 26.4 years and $15(42.8 \%)$ were in first pregnancy and $20(57.1 \%)$ were having one or more children before. The commonest complaint at presentation was pruritus (91.4\%). And the symptoms first appeared at 3436 weeks in 22 patients $(65.8 \%), 32-34$ weeks in 5 cases (14.4\%), before 32 weeks in 4 cases $(11.4 \%)$ and at 37 week in 3 patients (8.6\%). $15(42.8 \%)$ patients out of total 35 had delivered virginally while $20(57.2 \%)$ had cesarean delivery. Perinatal outcome were as follows 10 babies (28.5\%) had meconium stained liquor, 6 (17.1\%) had abnormal CTG pattern. While 6 (17.1\%) were diagnosed with intrauterine growth retardation and 9 babies (25.8\%) were born prematurely. One baby died in utero (2.8\%). ${ }^{17}$

A large retrospective study took place in Netherland in three hospitals from January 2005-August 2012 involving 215 women with cholestasis of pregnancy. The outcome variables were prematurity, meconium stained amniotic fluid, perinatal death and asphyxia and results showed that all mentioned complications are significantly higher in patients with cholystatis ${ }^{18}$ In India from November 2003 to November 2006 a study conducted in tertiary private hospital that also revealed higher incidence of pre-term birth and meconium staining. ${ }^{19}$ The fetal complication risks were significantly associated with serum bile acids levels and severity of cholestasis. ${ }^{20}$ Moreover latrogenic preterm delivery is five times higher in patients with diagnosed intrahepatic cholestasis. However some other studies found to have different results like $77 \%$ patients had preterm deliveries that were spontaneous deliveries and not iatrogenic. ${ }^{21}$ Similarly Geenes et al reported that frequency of prematurity in patients having singleton fetuses with severe ICP increased up-to 5.3 times when we compared it to compared to the singletons fetuses without $\mathrm{ICP}^{22}$ and the results of our study also affirm these results. Rook et al. reported contrary to the results of the previously mentioned studies. He described no association of biochemical markers to the increased fetal complications in ICP. ${ }^{23}$

In a few studies where there was no intrauterine fetal death, this finding can be attributed to early diagnosis and treatment using ursodeoxycholic acid and close monitoring. In patients with ICP the etiology of intra uterine fetal death is not obvious. However, theories suggest that this is linked to the toxic effects of SBAs. ${ }^{24}$ For our community if we want to reduce the fetal mortality we have to give proper attention to this serious health problem according to the level of complications it is associated with.

\section{CONCLUSION}

We conclude from the results of our study that some serous clinical recommendations should be made at each hospital and national levels to manage the complications of this serious problem. Mass awareness programs should be arranged at local and national levels using the media as well to generate general awareness among the population to seek medical help earlier in case of such complications as early intervention can prevent many of the complications.

Copyright $(12$ June, 2021.

\section{REFERENCES}

1. Mahajan N, Afzal A, Lone Ml. Outcome of pregnancy complicated by obstetric cholestasis: A prospective study. Int J Sci Stud. 2017; 5(3):271-4.

2. Karegoudar D, Patel RD, Dhital M, Amgain K. A study of liver disorder and its consequences in pregnant women with jaundice in tertiary care centre in Belgaum, Karnataka, India. J Dental Med Sci. 2014; 13(5):14-8.

3. Diken Z, Usta IM, Nassar AH. A clinical approach to intrahepatic cholestasis of pregnancy. Am J Perinatol. 2014; 31(1):1-8. 
4. Anjum N, Babar N, Sheikh S. Maternal and neonatal outcome in obstetric cholestasis: A comparison of early versus late term delivery. Pak Armed Forces Med J. 2015; 65(4):464-8.

5. Satia MN, Jandhyala M. A study of fetomaternal outcomes in cases of jaundice at a tertiary care centre. Int J Reprod Contracept Obstet Gynecol. 2016; 5:2352-7.

6. Geenes V, Chappell LC, Seed PT, Steer PJ, Knight M, Williamson C. Association of severe intrahepatic cholestasis of pregnancy with adverse pregnancy outcomes: A prospective population based case $\square$ control study. Hepatology. 2014; 59(4):1482-91.

7. Renu G, Nooren M, Abhilasha G, Neetu T, Vinita G, Rabinder I. Fetomaternal outcome in intrahepatic cholestasis of pregnancy. Sch J App Med Sci., 2017; 5(5A):1789-93.

8. Mishra N, Panigrahy S, Behera AA. Foetomaternal outcome of obstetric cholestasis. J. Evid. Based Med. Healthc. 2017; 4(59):3535-40.

9. D'Souza AS, Gupta G, Katumalla FS, Goyal S. Maternal and fetal outcome in liver diseases of pregnancy: A tertiary hospital experience. Int J Sci Res Publicat. 2015; 5(9):1-4.

10. Pokhrel S, Ghimire A, Jha GS, Chhetry M, Kumar M. Feto-maternal outcomes in intrahepatic cholestasis in pregnancy in a tertiary care centre in eastern Nepal. J Nobel Med Coll. 2016; 5:20-5.

11. Kondrackiene J, Beuers U, Kupcinskas L. Efficacy and safety of ursodeoxycholic acid versus cholestyramine in intrahepatic cholestasis of pregnancy. Gastroenterology 2005; 129:894-901.

12. Beuers U, Boyer JL, Paumgartner G. Ursodeoxycholic acid in cholestasis: Potential mechanisms of action and therapeutic applications. Hepatology. 1998 Dec. 28(6):1449-53.

13. Kretowicz E, Mclntyre HD. Intrahepatic cholestasis of pregnancy, worsening after dexamethasone. Aust $\mathrm{N}$ Z J Obstet Gynaecol. 1994 May. 34(2):211-3.
14. Beuers U. Drug insight: Mechanism and sites of action of ursodeoxycholic acid in cholestasis. Nat Clin Pract Gastroenterol Hepatol. 2006. 3:318-328.

15. Glantz A, Marschall HU, Lammert F, Mattsson LA. Intrahepatic cholestasis of pregnancy: A randomized controlled trial comparing dexamethasone and ursodeoxycholic acid. Hepatology. 2005 Dec. 42(6):1399-405.

16. Rook M, Vargas J, Caughey A, Bacchetti P, Rosenthal P, Bull L. Fetal outcomes in pregnancies complicated by intrahepatic cholestasis of pregnancy in a Northern California Cohort. PLoS One. 2012; 7(3): e28343.

17. Sohail S, Dar LR, Munawar S. Perinatal outcome in pregnancy complicated by obstetric cholestasis. Pak J Med Health Sci. 2016; 10(1):262-64.

18. Brouwers L, Koster M, Christian G, Kamperman H, Boom J, Evers I, et al. Intrahepatic cholestasis of pregnancy: Maternal and fetal outcomes associated with elevated bile acids. A JOG 2015; 212:101-7.

19. Padmaja M, Bhaskar P, Kumar G, Seetha R, Mahasweta C. A study of obstetric cholestasis. J Obstet Gynaecol India 2010; 60:225-31.

20. Saleh M, Abch KR. Intrahepatic cholestasis of pregnancy: Review of literature and evaluation of current evidence. J WomenHealth 2007; 16:833-41.

21. Demir BC, Güneş ES, Atalay MA. Intrahepatic cholestasis of pregnancy: Relationship between bile acid levels and maternal and fetal complications. Turk J Obstet Gynecol. 2014 Sep; 11(3): 148-152.

22. Geenes V, Chappell LC, Seed PT, Steer PJ, Knight M, Williamson C. Association of severe intrahepatic cholestasis of pregnancy with adverse pregnancy outcomes: A prospective population-based casecontrol study. Hepatology. 2014; 59:482-91.

23. Rook M, Vargas J, Caughey A, Bacchetti P, Rosenthal P, Bull L. Fetal outcomes in pregnancies complicated by intrahepatic cholestasis of pregnancy in a Northern California cohort. PLoS One. 2012; 7:28343.

24. Yıldırım G, Aslan H, Asar E, Gedikbası A, Güngordük K, Safak I, et al. Pregnancy outcome with intrahepatic cholestasis. J Turk Soc Obstet Gynecol 2007; 4(4):2503. 


\section{AUTHORSHIP AND CONTRIBUTION DECLARATION}

\begin{tabular}{|c|l|l|l|}
\hline No. & \multicolumn{1}{|c|}{ Author(s) Full Name } & \multicolumn{1}{|c|}{ Contribution to the paper } & Author(s) Signature \\
\hline 1 & Arooj Akram & Main Author \\
\hline 2 & Joveria Sadaf & Main AUthor \\
\hline 3 & Asia Aziz & Sana Ara & $\begin{array}{l}\text { Data analysis and proof } \\
\text { reading. } \\
\text { Data collection. }\end{array}$ \\
\hline 5 & Tanzila Rafiq & Data collection. \\
\hline 6 & Aslam Mahmood Malik & Result analysis \& discussion. & \\
\hline
\end{tabular}

